\title{
Questions spatiales et recherche numérique au Centre allemand d'histoire de l'art (DFK Paris)
}

Spatial Issues and Digital Research at the German Center for Art History (DFK Paris)

\section{Anne Klammt}

\section{OpenEdition}

\section{Journals}

Édition électronique

URL : https://journals.openedition.org/revuehn/1853

DOI : 10.4000/revuehn.1853

ISSN : 2736-2337

Éditeur

Humanistica

\section{Référence électronique}

Anne Klammt, «Questions spatiales et recherche numérique au Centre allemand d'histoire de l'art (DFK Paris) », Humanités numériques [En ligne], 3 | 2021, mis en ligne le 01 mai 2021, consulté le 16 juillet 2021. URL : http://journals.openedition.org/revuehn/1853 ; DOI : https://doi.org/10.4000/ revuehn. 1853

Les contenus de la revue Humanités numériques sont mis à disposition selon les termes de la Licence Creative Commons Attribution 4.0 International. 


\title{
humanités numériques
}

$3 \mid 2021$

Humanités numériques spatialisées

INFORMATION SPATIALE ET CORPUS TEXTUELS

Questions spatiales et recherche numérique au Centre allemand d'histoire de l'art (DFK Paris)

\author{
Spatial Issues and Digital Research at the German \\ Center for Art History (DFK Paris)
}

\section{Anne Klammt}

\section{Résumés}

Depuis 2014, le Centre allemand d'histoire de l'art (DFK Paris) dispose d'un nouveau département des humanités numériques, qui à la fois poursuit ses propres recherches et apporte son soutien aux autres départements du DFK Paris, en concevant et mettant en œuvre des projets de recherche numériques. Ces derniers ont permis de produire de vastes bases de données de référence et de proposer des modélisations d'espaces et de phénomènes spatiaux propres à chaque projet, comme le montrent les deux exemples que nous présentons. Les résultats obtenus offrent de nombreuses possibilités d'exploitation dans d'autres cadres de recherche.

Since 2014, the German Center for Art History Paris has had a department for Digital Humanities, which conducts its own research and supports projects at the DFK Paris by designing and implementing digital research. The projects have produced extensive data sets and have modeled space and spatial phenomena in different ways. Their results offer numerous opportunities for further research.

Seit 2014 hat das Deutsches Forum für Kunstgeschichte Paris eine Abteilung für Digital Humanities, die eigene Forschungen betreibt und die Projekte am DFK Paris bei der Konzeption und Umsetzung digitaler Forschungen unterstützt. Die Projekte haben umfangreiche 
Datenbestände vorgelegt und auf jeweils unterschiedliche Weise Raum und raumbezogene Phänomene in ihrer Untersuchung modelliert. Die Ergebnisse bieten zahlreiche Möglichkeiten weiterer Forschungen.

\section{Entrées d'index}

MOTS-CLÉS : histoire de l'art, humanités numériques spatialisées, TEI, corpus d'auteur, récit de voyage, gestion des données de la recherche

KEYWORDS: spatial digital humanities, art history, authorial corpus, TEI, travel literature, research data management

Le Centre allemand d'histoire de l'art à Paris (DFK Paris, pour Deutsches Forum Kunstgeschichte Paris) est membre de la fondation Max Weber, dont les dix instituts allemands en sciences humaines implantés à l'étranger ont pour vocation de renforcer les relations entre l'Allemagne et les États qui les accueillent. Le DFK Paris est le seul institut de la fondation ayant pour orientation l'histoire de l'art et pour mission d'" explorer l'art en France et les relations artistiques francoallemandes et de renforcer la collaboration dans le domaine des histoires de l'art allemande et française, y compris de ses partenaires dans le monde entier " (traduction de l'allemand; Max Weber Stiftung 2012). Toutes les recherches qui y sont réalisées sont donc liées à un espace donné ou recourent à la construction d'espaces du discours, recoupant et concernant des dimensions géographiques et constituant ainsi plus ou moins une histoire de l'art " française " et " allemande ". Dans de nombreuses études du DFK Paris, cette approche est complétée par ces objets de recherche (par exemple les œuvres d'art, les artistes, les ateliers, les musées) dans lesquels les espaces géographiques et les subdivisions territoriales jouent souvent un rôle.

Toutefois, les humanités numériques spatialisées ne constituent pas une priorité au DFK Paris, pas plus qu'elles ne sont une référence pour la plupart des chercheuses et chercheurs au moment de décider du sujet de leurs propres recherches, ou pour l'institut choisissant ses domaines de recherche. Pourtant de notre point de vue, les résultats des projets de recherche du DFK Paris fournissent des approches et données qui s'avèrent intéressantes pour les humanités numériques spatialisées. La présente contribution ${ }^{1}$ entend donc mettre l'accent sur ces dernières avec pour objectif de renforcer les échanges entre les différentes disciplines. Le DFK Paris peut aujourd'hui puiser dans sa longue expérience en conception et en accompagnement de projets de recherche en histoire de l'art numérique. Cette expérience repose sur le positionnement précoce du DFK Paris en faveur des humanités numériques. En effet, dès 2014, le DFK Paris crée un département d'humanités numériques, s'inscrivant ainsi comme l'un des précurseurs parmi les instituts de recherche en histoire de l'art de la sphère germanophone. Le DFK Paris a même été le premier des instituts réunis au sein de la fondation Max Weber à disposer d'un département d'humanités numériques. Jusqu'en 2019, c'est l'historien de l'art Thorsten Wübbena qui le dirigeait et le faisait 
constamment évoluer. Tous les projets numériques présentés ci-après ont été développés et mis en œuvre grâce à son étroite collaboration avec les chercheuses et les chercheurs du DFK Paris.

L'autrice de cette contribution a pris la direction du département des humanités numériques au printemps 2020. L'une de ses principales priorités consiste à poursuivre méthodiquement les projets développés jusqu'alors par le DFK Paris dans les domaines des technologies du Web sémantique, des standards, de la gestion des données de recherche et de la mise en réseau avec la community of interest. Les questionnements des données spatiales et géographiques survenant dans ce contexte s'avèrent particulièrement intéressants. Cela permet, d'une part, à l'histoire de l'art de bénéficier des expériences, ressources et infrastructures des autres disciplines et, de l'autre, de faire ses propres propositions et expériences dans le domaine des humanités numériques spatialisées. Selon l'autrice, l'histoire de l'art peut, par exemple, bénéficier de l'expérience de l'archéologie et des humanités classiques afin de constituer un index géographique basé sur la communauté scientifique, sur les données d'autorité et les offres de Linked Open Data. Tout cela peut être illustré par des exemples du Pelagios Network ${ }^{2}$, de Nomisma ${ }^{3}$ et Trismegistos ${ }^{4}$. Concernant les propositions spécifiques à l'histoire de l'art dans le champ des humanités numériques spatialisées, on peut attribuer à celles-ci un niveau conceptuel et un niveau pragmatique ${ }^{5}$. Parmi les sciences humaines, l'histoire de l'art est l'une des disciplines qui se préoccupe depuis très longtemps et de manière indépendante des objets en tant que composante de la perception et de la manifestation des espaces (intérieurs et extérieurs). En outre, l'étude des réseaux personnels et des régions de production, de distribution et de réception sont des domaines de recherche importants. Divers positionnements théoriques permettent de les interroger et de les observer sous un nouvel angle. Il existe donc des connaissances et approches propres à l'analyse qui sont reprises par les humanités numériques spatialisées (Besse 2010).

Sur un plan pragmatique, l'histoire de l'art est une fois de plus l'une de ces disciplines des sciences humaines dont le stock de médias et de données numériques s'accroît rapidement. Du matériel informatique, des logiciels et des normes sont nécessaires pour administrer, traiter présenter et récupérer ces supports et données. Les institutions du patrimoine culturel sont les moteurs de cette numérisation ${ }^{6}$. Les infrastructures existantes fournissent à leur tour une aide adaptée en mettant par exemple à disposition des thésaurus, tels que les Getty Vocabularies $^{7}$ et Iconclass ${ }^{8}$, mais aussi des formats permettant le recueil de métadonnées, tels que LIDO $^{9}$. La mise en œuvre rapide et généralisée du cadre international d'interopérabilité des images (IIIF ${ }^{10}$ ) par un grand nombre de bibliothèques, de musées et d'archives pour leurs propres archives numériques est une preuve impressionnante de l'importance que les institutions de recherche et les institutions du patrimoine culturel accordent au développement de solutions techniques, dans ce domaine. En outre, un certain nombre de clients de visualisation d'images, par exemple Openseadragon ${ }^{11}$ ou Mirador ${ }^{12}$, ont été développés pour répondre à leurs différents besoins. Cependant, le DFK Paris occupe un domaine différent dans le développement de solutions numériques destinées à l'histoire de l'art. Il les développe en réponse aux exigences de projets spécifiques. Ce faisant, ils s’inscrivent dans le prolongement 
des offres génériques et infrastructurelles mentionnées ci-dessus. À partir de 2014, l'accent a été mis sur la poursuite du développement de logiciels pour la gestion et l'enrichissement des bases de données d'images numériques ${ }^{13}$. Un autre domaine de travail a consisté à soutenir conceptuellement des projets de recherche de l'institut en utilisant des méthodes numériques. Deux projets qui ont modélisé la référence spatiale dans les données de différentes manières sont brièvement présentés ici. Tous deux fournissent donc des données et des approches pour des enquêtes plus approfondies avec une approche spécifique en humanités numériques spatialisées. 


\section{Projet ARCHITRAVE : modélisation des récits de voyages historiques}

Dans le cadre du programme ANR-DFG « Art et architecture à Paris et Versailles dans les récits de voyageurs allemands à l'époque baroque " (ARCHITRAVE), de nombreuses données sur des lieux géographiques, des sites historiques et des édifices de l'époque baroque en Allemagne, en France et aux Pays-Bas ont été extraites de six récits de voyageurs allemands rédigés entre 1685 et $1723^{14}$. Les auteurs de ces récits sont Christoph Pitzler (1657-1707), Ferdinand Bonaventure comte d'Harrach (1637-1706), Christian Friedrich Gottlieb von dem Knesebeck (avant 1680 - après 1727), Leonhard Christoph Sturm (1669-1719), Lambert Friedrich Corfey (1668-1733) et son frère Christian Heinrich Corfey (1670-1652) et enfin Johann Balthasar Neumann (1687-1753). Les données extraites de ces six textes permettent de reconstruire les itinéraires des visiteurs et de découvrir les espaces géographiques et culturels qui ont retenu leur attention entre la fin $d^{u}$ XVII $^{\mathrm{e}}$ et le début du XVIII ${ }^{\mathrm{e}}$ siècle. Le projet $A R$ CHITRAVE comporte plusieurs volets, dont l'édition numérique de ces six manuscrits. Les descriptions des lieux et des édifices, incluant les intérieurs de bâtiments visités à Paris et à Versailles, constituent le cœur du projet. Afin de permettre à la communauté internationale des historiens de l'art d'avoir accès aux textes, les six manuscrits sont publiés en allemand avec leur traduction française. Les deux versions ont été respectivement encodées en XML-TEI et c'est l'environnement collaboratif TextGrid Lab $^{15}$ qui a été utilisé pour produire l'édition numérique. Tous les fichiers XML ainsi que le schéma d'encodage TEI sont en libre accès et téléchargeables au sein du TextGrid Repository ${ }^{16}$. Le portail d'édition numérique ARCHITRAVE ${ }^{17}$ proposera une vue synoptique des manuscrits édités permettant d'afficher les fac-similés en vis-à-vis de la transcription et de la traduction. Sa mise en ligne aura lieu en $2021^{18}$. Le portail proposera en outre une carte interactive permettant de visualiser les itinéraires des voyageurs en Europe et en France ainsi que les lieux visités à Paris. Les lieux et les édifices visités étant particulièrement importants dans ce projet, nous proposons de nous intéresser à la modélisation et à la mise en œuvre des données. Au cours du projet, les chercheurs ont élaboré une modélisation en trois étapes : d'abord un balisage TEI assez simple des entités nommées (encodage des lieux, des personnes et des œuvres cités dans les textes), puis l'intégration de ces entités nommées dans trois « registres » (lieux, personnes, œuvres) se présentant dans des tableaux organisés par champs et colonnes de données, et enfin l'alimentation et l'enrichissement de ces registres par du contenu scientifique et des liens vers des référentiels existants. Cette méthode a permis d'utiliser un balisage TEI relativement simple, mais avant tout de démarrer le travail d'encodage des textes originaux et des traductions assez tôt dans le projet, alors que la majorité des textes n'avaient jamais été publiés auparavant et que, par conséquent, les chercheurs n'avaient pas pris toute la mesure de leur complexité. Cette approche a aussi permis d'apporter des enrichissements sur les entités nommées sans avoir à modifier les fichiers XML-TEI puisque l'information était saisie uniquement dans les registres. 
Dans un premier temps, les lieux ont été encodés à l'aide de deux balises : < placeName > et <geogName $>$. Tous les noms de lieux (hors noms topographiques et hydrographiques) et d'édifices ont été encodés avec la balise $<$ placeName $>$. Il en résulte un ensemble de données d'une grande disparité, allant des villes aux quartiers, en passant par les édifices, les parcs, les fontaines et les obélisques. Grâce à l'encodage de toutes ces entités, les historiens de l'art pourront entre autres étudier la réception critique des édifices et des œuvres à l'époque baroque.

À l'intérieur de la catégorie "Places ", les éléments ont été différenciés en utilisant six «types" (continent, country, region, district, city, place) et trois "sous-types" (passingBy, rooms, residences). Dans trois des six manuscrits (Corfey, Harrach et Knesebeck), les étapes de voyage en Europe et en France ont été encodées dans les fichiers XML-TEI à l'aide de la balise < placeName> associée au " type " city ou place et au sous-type passingBy et sont dotées d'un identifiant unique, pour les distinguer des autres lieux auxquels les voyageurs font référence dans les textes. Pour certaines de ces étapes, les voyageurs ont par ailleurs décrit certains édifices, bâtiments, parcs et jardins qui s'y trouvaient. Ces lieux décrits sont encodés avec la balise TEI < placeName> et le type place et sont dotés d'un identifiant ${ }^{19}$. Les monuments (mausolées, tombeaux, fontaines, obélisques...) ainsi que les œuvres d'art présentes dans les espaces intérieurs (peintures, sculptures, objets d'art...) sont encodés en tant que artworks (œuvres). L'encodage seul ne donne pas d'informations sur les relations entre les entités nommées. Ce sont les registres qui permettent d'établir les liens entre ces dernières : par exemple, la notice d'une œuvre d'art citée comporte un champ "Localisation ancienne " (c'est-à-dire la localisation de l'œuvre à l'époque des voyageurs) avec un lien vers la notice "place » associée, puisque le lieu où se trouve l'œuvre citée est mentionné dans le texte. Bien que d'un intérêt moindre pour l'équipe, les noms géographiques (<geogName $>$ ) ont été encodés avec six sous-types (forest, mountain, water, desert, island, plains).

Les termes encodés avec les balises < placeName > ou <geogName > se trouvent dans le registre des lieux, auxquels on a attribué un " type » correspondant à une " catégorie " de lieu ; ces catégories sont les suivantes : lieux géographiques, édifices publics, édifices domestiques, édifices domestiques (intérieurs), édifices religieux, monuments, constructions militaires, parcs et jardins, autres. Par ailleurs, 1189 entités sur 1333 ont été géolocalisées manuellement (extraction des coordonnées géographiques à partir de Google Maps). Afin d'harmoniser la saisie dans le registre, un glossaire franco-allemand a été mis en place. 


\begin{tabular}{|c|c|c|}
\hline \multirow{6}{*}{ Registre "Places" } & hen vers un référentiel & Wikidata item: $Q 3145767$ \\
\hline & coordonnés géographiques & WGS 84: 48.859500, 2.339700 \\
\hline & \multirow[t]{3}{*}{$\begin{array}{l}\text { informations renseignees par } \\
\text { les chercheurs }\end{array}$} & 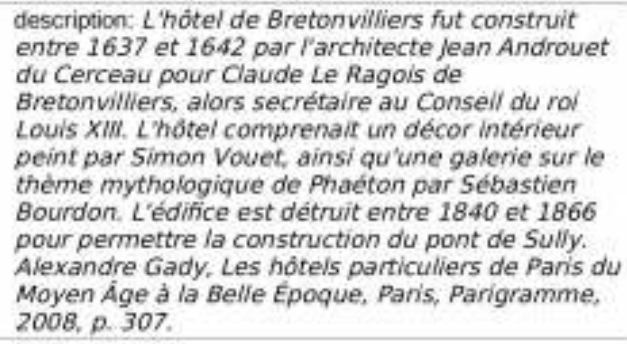 \\
\hline & & $\begin{array}{l}\text { date construction/destruction: hötel particulier: } \\
\text { bureau et imprimenle de la ferme générale de } \\
1719 \text { à } 1789\end{array}$ \\
\hline & & état détruit \\
\hline & classification ( 10 'types') & type: domestic \\
\hline Document XML-TEI & encodage & $\begin{array}{l}\text { <placeName ref="plc.textgnid:3ptfg" type="place"s Hôtel } \\
\text { de Bretonviliers</placeName> }\end{array}$ \\
\hline
\end{tabular}

Image produite par l'autrice

La modélisation des données ressemble à une structure de Linked Data (données liées du Web des données ou Web sémantique), dans laquelle différents environnements sémantiques sont construits à partir des entités individuelles. Contrairement à d'autres projets où l'encodage TEI des entités nommées peut être plus complexe, ici l'équipe a choisi de reporter la complexité des informations au sein des registres (alignement sur un référentiel, qualification de chaque lieu en s'appuyant sur une ontologie propre [les types du registre "Places"], description documentée par une source pour une sélection de lieux importants). L'enrichissement sémantique reste ainsi flexible et a pu se faire en plusieurs étapes, sans pour autant modifier l'annotation TEI. Avec ces registres ou index des noms de lieux, personnes et œuvres d'art du début du XVIII ${ }^{\mathrm{e}}$ siècle, le projet offre une ontologie ainsi qu'un vocabulaire contrôlé réutilisables par d'autres projets de recherche ${ }^{20}$. Les perspectives de recherche ultérieures autour des six manuscrits du projet ARCHITRAVE consisteraient principalement dans l'élaboration de la microtopographie et d'un travail sur les intérieurs du château de Versailles ${ }^{21}$. 


\section{Projet OwnReality : modélisation numérique des expositions en tant qu'événements spatiotemporels}

Le projet de recherche "OwnReality. À chacun son réel. La notion de réel dans les arts plastiques en France, RFA, RDA et Pologne entre 1960 et 1989 " a étudié la façon dont les notions de réel et de réalité ont été utilisées dans les discours sur l'art en Europe de l'Est et de l'Ouest pendant la guerre froide. Hébergé par le Centre allemand d'histoire de l'art Paris entre 2011 et 2016 et dirigé par Mathilde Arnoux, le projet a été financé par une bourse Starting Grant du Conseil européen de la recherche $\left(\mathrm{ERC}^{22}\right)$.

Parmi les divers résultats du projet, nous nous intéresserons ici à une base de données plurilingue (français, allemand, anglais et ponctuellement polonais). Elle rassemble notamment 2560 articles tirés de 16 revues d'art spécialisées, dépouillées par l'équipe de recherche, et des données structurées concernant 1478 expositions qui se sont tenues entre 1960 et 1989 dans les quatre pays. Articles et expositions font chacun l'objet d'un commentaire. La base de données inclut également la transcription d'entretiens menés dans le cadre du projet avec des acteurs de l'époque étudiée (artistes, galeristes, historiens de l'art d'Allemagne, de France et de Pologne), ainsi que des études de cas analysées par les chercheuses et chercheurs associés au projet. Les noms d'auteurs, de personnes, de villes, de pays, de lieux d'expositions, de revues, de partis et d'associations mentionnés dans ces différents éléments, ont été indexés, une série de notions (groupes/tendances/idées et techniques/genres/médium) y a par ailleurs également été rattachée. La base de données est disponible en ligne ${ }^{23}$, au format HTML pour la plupart des contenus. Toutes les entrées sont liées entre elles. Des recherches croisées peuvent y être effectuées en plein texte ou au moyen de motsclés classés en dix catégories correspondant à l'indexation des données. Cet ensemble de mots-clés, ainsi que les liens internes, ont été établis de manière spécifique au projet. Le DFK Paris propose par ailleurs un accès libre aux données au format JSON ${ }^{24}$.

Du point de vue des données, les quelque 1500 expositions enregistrées sont particulièrement intéressantes pour ce qui nous concerne ici. Les expositions sont en effet des entités qui se laissent délimiter à la fois dans l'espace et dans le temps. Elles peuvent, par exemple, se dérouler de manière récurrente dans des lieux spécifiques, être successivement présentées sous forme itinérante dans divers lieux, ou être réparties sur différents sites et à différentes dates. Des phénomènes comparables ont été récemment désignés, par la modélisation d'ontologies pour la recherche archéologique, comme des volumes spatiotemporels (space time volumes) et modélisés comme une extension spécifique de l'ontologie CIDOC CRM ou comme un index temporel. Pour le projet de recherche OwnReality, les expositions ont constitué par ailleurs l'un des objets d'étude cardinaux. En plus de réunir des œuvres, des artistes, des conservateurs, des commissaires, des critiques et des visiteurs, elles servent également de nœud dans la modélisation du matériel de re- 
cherche : elles sont recensées dans les articles des revues dépouillées, elles sont au cœur des entretiens avec des témoins contemporains ou elles font l'objet des études de cas rédigés par les chercheurs.

Dans la base de données du projet OwnReality, les expositions sont donc conçues comme des entités caractérisées par des attributs spatiaux et temporels. D'autres attributs s'y ajoutent ensuite, relatifs aux personnes qui y ont participé à différents titres (artiste, commissaire, conservateur) ou qui se sont exprimées à leur sujet dans des articles publiés dans les revues dépouillées (critique), ou encore relatifs aux notions pertinentes pour la recherche en histoire de l'art (groupes/tendances/idées et techniques/genres/médium). L'entité " exposition » est donc constituée par un titre et un ensemble d'attributs. Les caractéristiques spatiales de l'événement concernent non seulement le lieu, la ville et le pays où l'exposition a été présentée, mais elles s'appliquent aussi à d'autres particularités pertinentes d'un point de vue thématique, que ce soit la nationalité ou le pays d'origine d'un artiste exposé, d'un commissaire ou d'un critique, l'État auquel sont rattachés l'institution, le parti ou l'association à l'origine de son organisation, ou le lieu de publication des revues qui en ont rendu compte.

Le référencement de coordonnées de géocodage et leur couplage avec un index géographique (gazetteer) ne se sont pas révélés pertinents dans le cadre de l'analyse poursuivie par ce projet. Néanmoins, il serait envisageable de considérer un enrichissement en ce sens, puisque l'équipe de recherche a standardisé les références des lieux, institutions, personnes, de la date, ainsi que d'autres facettes, sur la base de vocabulaires internes contrôlés. Les quelque 1500 expositions saisies et modélisées constituent ainsi, en tant qu'événements spatiotemporels reliés à des personnes et publications, une ressource considérable pour les humanités numériques spatialisées.

Le DFK Paris a commencé un premier enrichissement des données à l'automne 2020 en référençant tous les auteurs selon les données d'autorité de la GND (Gemeinsame Normdatei, gérée par la Bibliothèque nationale allemande ${ }^{25}$ ) et celles appliquées par la Bibliothèque nationale de France $^{26}$. De la même manière que pour le projet ARCHITRAVE précédemment décrit, il est prévu de mettre les lieux d'exposition en conformité avec le Getty Thesaurus of Geographic Names ${ }^{27}$. Ces initiatives sont liées à un transfert de la base de données en vue de son stockage dans le dépôt de données de recherche heiDATA de la bibliothèque universitaire de Heidelberg ${ }^{28}$. La publication des expositions sous forme de données ouvertes liées (Linked Open Data), voire d'un index temporel (time gazetteer), est une autre perspective possible. 


\section{Perspectives des humanités numériques spatialisées au DFK Paris}

Dans les projets numériques du DFK Paris les questionnements sur l'espace jouent souvent un rôle, mais celui-ci est généralement plutôt discret, voire caché. En ce moment, le département des humanités numériques poursuit la mise en place d'infrastructures numériques adaptées aux besoins concrets des projets de recherche. Cela inclut la prise en compte d'un référentiel des données de recherche afin de les sécuriser et de mieux les gérer. Eu égard aux besoins des humanités numériques spatialisées, il convient d'utiliser des formats de données accessibles et ouverts (par exemple, KML, GeoJSON ou GeoTIFF ${ }^{29}$ ) pour la sécurisation dans le référentiel, sous réserve que les contenus des projets correspondants aient pu être traités. Une procédure étroitement liée à cette étape consiste à rendre les données interopérables sur un plan sémantique et à faciliter l'exploitation des gisements de données disponibles grâce à l'enrichissement sémantique au moyen des données d'autorité, par l'alignement avec des ontologies (data mapping) et par la mention des métadonnées utiles. Cela signifie qu'il faut continuer de soutenir et promouvoir la prise en compte des index géographiques pertinents et aussi de Wikidata.

Comme l'illustrent les présentes études de cas, la mise en place et le suivi des vocabulaires contrôlés au sein des projets numériques font souvent partie du travail de fond. Les listes de vocabulaire bénéficient donc de l'expertise des équipes. Elles représentent souvent très exactement un champ de recherche spécifique. L'élaboration de vocabulaires contrôlés ne constitue pas seulement une performance réalisée en vue de ces projets mais aussi une base pour des recherches à venir, qui pourra être fusionnée avec d'autres bases de connaissances. L'objectif à moyen terme est donc de mettre à disposition le vocabulaire des projets numériques du DFK Paris sous forme de Linked Open Data. Cependant, pour pouvoir réutiliser ces "données liées ouvertes ", leur mise à disposition n'est pas la seule condition requise : il faut aussi qu'elles se réfèrent à des thésaurus qui soient pertinents pour les chercheurs (Piotrowski et al. 2014).

\section{Bibliographie}

Architrave. 2019. « ARCHITRAVE Schema_v7 ». https://hdl.handle.net/21.11113/0000-000 C-4F5B-7.

Besse, Jean-Marc. 2010. "Approches spatiales dans l'histoire des sciences et des arts ". L'Espace géographique 39 (3) : 211-224. https://doi.org/10.3917/eg.393.0211.

Deutsches Forum für Kunstgeschichte. 2017. "Jahresbericht des Deutschen Forums für Kunstgeschichte Paris 2015/2016 - Rapport d'activité du Centre allemand d'histoire de l'art 2015/2016 ». Paris : Deutsches Forum für Kunstgeschichte Paris. https://doi.org/10.115 88/dfkjb.2015.0.

Deutsches Forum für Kunstgeschichte. 2018. «Jahresbericht des Deutschen Forums für Kunstgeschichte Paris 2016/2017 - Rapport d'activité du Centre allemand d'histoire de l'art 2016/2017 ». Paris : Deutsches Forum für Kunstgeschichte Paris. https://doi.org/10.115 88/dfkjb.2016.0. 
Deutsches Forum für Kunstgeschichte. 2019. «Jahresbericht des Deutschen Forums für Kunstgeschichte Paris 2017/2018 - Rapport d'activité du Centre allemand d'histoire de l'art 2017/2018 ». Paris : Deutsches Forum für Kunstgeschichte Paris. https://doi.org/10.115 88/dfkjb.2017.0.60934.

Deutsches Forum für Kunstgeschichte. 2020. «Jahresbericht des Deutschen Forums für Kunstgeschichte Paris 2018/2019 - Rapport d'activité du Centre allemand d'histoire de l'art 2018/2019 ». Paris : Deutsches Forum für Kunstgeschichte Paris. https://doi.org/10.115 88/dfkjb.2018.0.

Max Weber Stiftung. 2012. "Satzung der Max Weber Stiftung - Deutsche Geisteswissenschaftliche Institute im Ausland (ab 1. Juli 2012 geltende Fassung) ». https://www.maxweberstiftung.de/fileadmin/user_upload/upload/Satzung_MWS_Stand_1.7.2012.pdf.

Piotrowski, Michael, Giovanni Colavizza, Florian Thiery et Kai-Christian Bruhn. 2014. « The Labeling System : A New Approach to Overcome the Vocabulary Bottleneck ». Dans DH-CASE II : Collaborative Annotations on Shared Environments: Metadata, Tools and Techniques in the Digital Humanities, 1-6. Fort Collins : Association for Computing Machinery. $\mathrm{h}$ ttps://doi.org/10.1145/2657480.2657482.

Tarver, Hannah. 2012. « Controlled Vocabulary Graphic ». Image. « Controlled Vocabularies for Digital Collections Workshop ", The Humanities and Technology Camp (THATCamp), 2012, Denton, Texas, United States. 22 septembre. https://digital.library.unt.edu/ark:/67531/metadc111289/.

\section{Notes}

1 L'autrice remercie Mathilde Arnoux et Thomas Kirchner (DFK Paris), Markus Matoni (Staats- und Universitätsbibliothek Göttingen), Alexandra Pioch (Centre de recherche du château de Versailles) et Thorsten Wübbena (Leibniz-Institut für Europäische Geschichte Mainz) pour leur aide et leurs conseils.

2 https://pelagios.org.

3 http://nomisma.org.

4 https://www.trismegistos.org.

5 L'autrice aimerait expliquer ici qu'en tant qu'archéologue de formation, elle peut dans une large mesure faire une description du niveau pragmatique.

6 La Bibliothèque nationale de France ainsi que sa bibliothèque numérique Gallica (https: //gallica.bnf.fr) jouent un rôle crucial en France ; en Allemagne, le Museum für Kunst und Gewerbe Hamburg (https://www.mkg-hamburg.de/en/) est un véritable pionnier et le Rijksmuseum à Amsterdam bénéficie d'une grande considération dans le monde depuis longtemps (https://www.rijksmuseum.nl/en/) ; Europeana occupe une place importante en tant que plateforme de données pour les instances européennes des GLAM (Galleries, Libraries, Archives and Museums) (https://www.europeana.eu/en/).

7 Le Getty Art and Architecture Thesaurus est un vocabulaire contrôlé, qui est édité par le Getty Trust. Le contrôle éditorial est donc assuré par le Getty Research Institute. http://w ww.getty.edu/research/tools/vocabularies/index.html.

8 Iconclass est une classification de bibliothèque spécialisée conçue pour l'art et l'iconographie, qui est maintenue par le Rijksbureau voor Kunsthistorische Documentatie (Bureau national pour la documentation relative à l'histoire de l'art). http://www.iconclass.or g.

9 LIDO (Lightweight Information Describing Objects) est un format permettant de fournir des informations de collecte pour la découverte de ressources. LIDO est soutenu par un groupe de travail de l'ICOM (Comité international pour la documentation). http://network.icom.museum/cidoc/working-groups/lido/what-is-lido/.

10 International Image Interoperability Framework : https://iiif.io.

11 https://openseadragon.github.io/examples/tilesource-iiif/.

12 https://projectmirador.org.

13 Logiciel pour la banque d'images KOR as a service (https://dfk-paris.org/fr/research-pr oject/kor-service-713.html) et les projets connexes Wikidata x ConedaKOR (https://dfk-pa ris.org/fr/research-project/wikidata-X-conedakor-2047.html) et similARiTy (https://dfk-p aris.org/fr/research-project/similarity-2277.html). À propos du contexte concernant les 
projets, cf. Deutsches Forum für Kunstgeschichte (2017 : 96) ; Deutsches Forum für Kunstgeschichte (2018: 88) ; Deutsches Forum für Kunstgeschichte (2019 : 98-101) ; Deutsches Forum für Kunstgeschichte (2020 : 80-83).

14 Le projet ARCHITRAVE est un partenariat entre l'université de Marbourg (direction), le Centre de recherche du château de Versailles, le DFK Paris et la Niedersächsischen Staatsund Universitätsbibliothek Göttingen. Le projet bénéficie du soutien de l'Agence nationale de la recherche (ANR) et de la Deutsche Forschungsgemeinschaft (DFG).

15 TextGrid Lab (https://textgrid.de/en/)constitue un environnement de recherche virtuel qui, en tant que logiciel open source, est adapté aux chercheuses et chercheurs en sciences humaines et sociales pour l'édition de leurs textes. Les éditions numériques sont sauvegardées dans le TextGrid Repository associé au TextGrid Lab.

16 Les images numérisées des manuscrits, les schémas de données, le Schematron, les documents TEI et autres données sont en accès libre au sein du TextGrid Repository (voir https://textgridrep.org, « Projekt Architrave »).

17 https://architrave.eu.

18 La conception technique et la mise en œuvre de l'édition numérique sont réalisées par la Staats- und Universitätsbibliothek Göttingen, et plus précisément par Markus Matoni et Christoph Kudella.

19 Pour le schéma TEI, voir Architrave (2019).

20 L'autrice n'utilise pas les notions d'ontologie et de vocabulaire contrôlé au sens d'unités sélectives, comme le fait Tarver (2012), mais comme quelque chose de subdivisible en fonction de sa complexité.

21 Pour ce faire, différents travaux préliminaires ont été menés par le projet. De plus amples informations sont disponibles auprès de la direction du projet (cf. https://architrave.eu, onglet " contact»).

22 Plus d'informations sur https://dfk-paris.org/fr/ownreality/.

23 https://dfk-paris.org/fr/page/ownrealityrecherche-croisee-1353.html.

24 https://github.com/dfk-paris/own-reality/.

25 https://www.dnb.de/EN/Professionell/Standardisierung/GND/gnd_node.html.

26 https://data.bnf.fr/ et https://www.bnf.fr/fr/donnees-autorite-bnf.

27 http://www.getty.edu/research/tools/vocabularies/tgn/.

28 https://heidata.uni-heidelberg.de.

29 Standards ouverts, cf. https://www.ogc.org/standards/community.

\section{Auteur}

\section{Anne Klammt}

Deutsches Forum für Kunstgeschichte Paris - Centre allemand d'histoire de l'art, Paris, France

Anne Klammt est responsable de la recherche numérique au Centre allemand d'histoire de l'art à Paris depuis le mois de mars 2020.

ORCID 0000-0003-3697-9241

aklammt@dfk-paris.org

\section{Droits d'auteur}

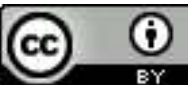

Les contenus de la revue Humanités numériques sont mis à disposition selon les termes de la Licence Creative Commons Attribution 4.0 International. 